\title{
Karol Karp
}

https://orcid.org/0000-0002-9449-8297

(Toruń)

VIAGGIARE E VIVERE ALTROVE.

SULLA CONDIZIONE DEL MIGRANTE NEL PAESE

D'ADOZIONE NEL ROMANZO „M” DI RON KUBATI

\begin{abstract}
The aim of the article is to present the existential status of a migrant on the basis of Ron Kubati's novel "M", and to define the features of the phenomenon of migration. The analysis focuses on two areas. First, it shows the major problems which determine the character's internal state, namely lack of knowledge about the new environment and the hostility of the local community. Second, it outlines the positive aspects of staying there, highlighting the dominant ones.

\section{Key words}

Ron Kubati, migration, migrant in the new reality, existential status, problems, integration process
\end{abstract}




\section{INTRODUZIONE}

Sono circa una quarantina gli scrittori di origine albanese e senz'altro rivestono un ruolo importante nel panorama della letteratura italiana della migrazione. La loro importanza non è però determinata soltanto dal criterio quantitativo, ma soprattutto dal valore letterario delle opere che hanno prodotto, attestato da più riconoscimenti. Ad esempio nel 2008 la Fondazione Carical Grinzane Cavour ha onorato Elvira Dones per il romanzo „Vergine giurata” (2007). Lo stesso premio ha ottenuto anche Ornela Vorpsi con l'opera "Il paese dove non si muore mai” (2005). Kubati invece ha vinto il concorso Popoli in cammino. La giuria ha apprezzato il suo testo proposto in versione inedita, poi pubblicato nel 2007, sotto il titolo „Il buio del mare”. Con lo stesso testo l'autore è giunto in semifinale allo Strega nel $2008^{1}$.

Nato a Tirana nel 1971 da una famiglia di dissidenti comunisti, Kubati si reca in Italia all'inizio degli anni Novanta dopo il crollo della dittatura ${ }^{2}$. Attualmente vive negli Stati Uniti e continua a pubblicare in italiano ${ }^{3}$. La sua produzione $e^{4}$ è in sintonia con quella degli altri autori migranti di origine albanese $^{5}$. Uno dei principali punti di convergenza è costituito dalla presenza dell'autobiografismo, individuabile nei riferimenti sia al paese natale sia

\footnotetext{
${ }^{1}$ Gli elementi forti delle opere di Kubati, che di sicuro hanno contribuito al suo successo letterario, sono la lingua e lo stile. Il romanzo „M” è dotato di una lingua scorrevole e precisa che trasmette perfettamente i pensieri e le idee dei personaggi. Lo scrittore usa abbastanza spesso parole inglesi, forse perché in genere sono molto diffuse nell'italiano contemporaneo, che ha imparato dal vivo. Con sorpresa non abbiamo trovato parole albanesi, di solito presenti nei testi di autori migranti provenienti dall'Albania. Esse sono facilmente rintracciabili in opere di Artur Spanjolli come „La Teqja” (2006) oppure „La sposa rapita” (2011). Lo stile di Kubati è sintetico, piuttosto asciutto e tale sua caratteristica lo rende simile per esempio a quello di Vorpsi.

${ }^{2}$ Bregola 2002, p. 28.

${ }^{3}$ Il fatto fa venire in mente l'esperienza migratoria di Dones. La scrittrice ha abbandonato il paese d'origine verso la fine degli anni Ottanta, ha trascorso qualche anno nella Svizzera italiana e si è trasferita negli Stati Uniti. Da poco tempo è ritornata in Svizzera. Vista la storia di Dones e Kubati, lo studioso può avere dubbi sui criteri da applicare alla loro classificazione. Gli autori possono essere considerati rappresentanti della letteratura italiana della migrazione ad esempio per la lingua della loro espressione letteraria, per le esperienze migratorie che vivono e che consentono loro di conoscere nuovi spazi e nuove culture. Quello che li differenzia da molti scrittori migranti è il fatto che attualmente non vivono in Italia; occorre aggiungere che non vi vive neppure Vorpsi. Tuttavia è possibile che in futuro rimangano stabilmente proprio nel paese la cui lingua padroneggiano perfettamente.

${ }_{4}^{4}$ Oltre al romanzo in esame, Kubati ha pubblicato in italiano le seguenti opere: „La vita dell'eroe” (2016), „Il buio del mare” (2007), „Va e non torna” (2000), „Tra speranza e sogno” (1992), „Venti di libertà e gemiti di dolore” (1991).

${ }^{5}$ Tra i più importanti autori migranti italofoni di origine albanese, non menzionati ancora nel presente articolo, si devono annoverare: Leonard Guaci, Anilda Ibrahimi e Darien Levani.
} 
all'esperienza migratoria che hanno vissuto. Nell'ultimo romanzo di Kubati intitolato „La vita dell'eroe” gli elementi autobiografici si possono scorgere in una ricca visione dell'Albania, che ingloba i tempi che vanno dall'occupazione fascista fino all'epoca del comunismo ${ }^{6}$. Nell'opera che ci interessa in questa sede invece essi sono inerenti alla sua permanenza in Italia.

Lo scopo del presente articolo è quello di analizzare tutti gli aspetti più importanti della condizione del migrante che ha abbandonato la terra natale e di determinare il carattere del fenomeno della migrazione. La disamina viene organizzata in due parti. Nella prima parte l'attenzione è focalizzata sui problemi cui il protagonista è costretto a fare fronte, la seconda parte invece è dedicata al processo della sua integrazione.

\section{NEL NUOVO SPAZIO VITALE: PROSPETTIVA CONFLITTUALE}

Con la narrazione in prima persona, che avvicina in modo naturale la storia raccontata alla figura dell'autore ${ }^{7}$, ci inoltriamo nel quadro della vita del protagonista, il cui nome non viene svelato. $\grave{E}$ un individuo che vive nel paese d'accoglienza e vi affronta difficoltà che si estendono a due ambiti: quello fisico e quello umano. I motivi precisi del suo arrivo non sono esplicitamente trasmessi e tale tecnica, occorre dirlo, risulta piuttosto rara nel corpus classificato come letteratura italiana della migrazione. Di solito l'autore informa subito il lettore del perché della partenza del migrante per uno stato straniero, molto spesso motivata da considerazioni economiche e politiche. A questo punto vanno citati alcuni esempi. Nell'opera „I nipoti di Scanderbeg" (2012) di Spanjolli, gli albanesi fuggono in Italia in quanto stanchi della vita nella realtà distrutta dal comunismo e desiderosi di trovarne un'altra migliore. Nel romanzo „Voglio un marito italiano. Dall'est per

\footnotetext{
${ }^{6}$ Kubati pone un accento particolare sui cambiamenti che hanno luogo nell'Albania del Novecento, rileva come da stato feudale, religioso e conservatore essa si sia trasformata in quello profano, sottomesso totalmente al dittatore. Il protagonista Sami Keçi partecipa attivamente a vari avvenimenti storici e politici, è membro della resistenza partigiana durante l'occupazione fascista, poi quella nazista, per diventare finalmente un personaggio importante nei servizi di Enver Hoxha. Durante la guerra lotta per un ideale che considera dignitoso e non esita a rischiare la propria vita. Un elemento interessante della trama costituiscono le sue relazioni con le donne che lo portano a vivere momenti sia felici che infausti.

${ }^{7}$ Il personaggio principale è l'alter ego dello stesso Kubati, che dopo l'arrivo in Italia ha dovuto fronteggiare varie difficoltà, tra cui la ricerca di un editore che fosse interessato a pubblicare i suoi libri.
} 
amore?" (2006) di Marina Sorina il personaggio femminile lascia l'Ucraina dove dominano la povertà e la disoccupazione. In ambedue i testi spicca il dolore interiore dei migranti. Ne „I nipoti di Scanderbeg” molti di loro sono rifiutati dalle autorità, maltrattati dagli ufficiali e rimpatriati. Sorina invece denuncia la sofferenza di giovani donne dell'Europa orientale esposte a comportamenti gretti da parte degli uomini italiani, rilevando come non siano intenzionati che a sfruttarle sessualmente.

$\mathrm{Al}$ protagonista di „M” la nuova realtà provoca paura e senso di smarrimento; ad un tratto si trova davanti a spazi ignoti che è costretto a esplorare, ogni tanto lo fa anche spontaneamente.

Molto spesso mi perdevo. Capito anche quel pomeriggio. Intorno a me tutto era
estraneo: gli edifici, le strade, le persone, le auto, il cielo chiuso e pesante come
i miei piedi e la mia testa. Il buio avanzava. (...) I marciapiedi si davano il cambio,
le auto giravano, i grattacieli mi circondavano. Disperatamente in cerca di punti
fermi, di elementi familiari. Camminavo, ma era come se non lo facessi. Mi
allontanavo non so da cosa, per avvicinarmi non so dove.

Il migrante si perde di continuo e perciò può essere paragonato a un bambino che scopre passo dopo passo la topografia della città in cui vive. Nella propria interiorità è di sicuro sospeso tra il ricordo del paese d'origine, che conosce bene, e lo spazio dove tutto gli pare strano, compresi i locali in cui si imbatte. Occorre dire che ai loro occhi lui stesso si configura come un altro, è esposto a vari atti di intolleranza, a situazioni in cui si sente segnato dalla provenienza.

L'agente gli restituì i documenti. A me dispiacque. Il barbone aveva un'aria di sfida che mi dava sui nervi. Adesso toccava a me.

- Lei è straniero? Eppure non sembra... - mormorò il più basso.

$-\mathrm{Si}$.

- Girati. Allarga le gambe.

Mi sentii afferrare dalla giacca e costretto ad appoggiare le mani sul vetro. I due mi perquisirono. La gente mi guardava male. (...) Intorno a me si formò un cerchio. Tutti, tranne il professore, tenevano d'occhio me. (...) La perquisizione terminò senza alcun risultato. Gli agenti si allontanarono senza dire una parola ${ }^{9}$.

L'episodio succitato attesta come le origini determinino la percezione del soggetto migrante e generino trattamenti ingiusti e umilianti. Vi campeggiano il suo dolore interiore e il senso di inferiorità. Da un lato Kubati intende denunciare la sorte deplorevole dello straniero che vive in Italia, dall'altro si pronuncia sugli autoctoni, non di rado fedeli agli stereotipi e ai pregiudizi, indaga anche su altri importanti aspetti della realtà del paese. Sottolinea il suo

${ }^{8}$ Kubati 2002, p. 23.

${ }^{9}$ Ibidem, p. 12. 
carattere multiculturale costituito da una mescolanza di religioni, credenze e tradizioni. Nel testo si possono notare riferimenti alla difficile situazione economica di alcuni locali, di frequente privi della possibilità di trovare lavoro e propensi a commettere diversi reati, nonché al fenomeno della prostituzione. In questo contesto vale la pena accennare al fatto che molti fenomeni sociali negativi, compresa l'esistenza della criminalità organizzata, in cui sono coinvolti gli stessi italiani, ma anche gli albanesi, si percepiscono nel romanzo „Toringrad” (2016) di Darien Levani, uno scrittore migrante, nato a Fratar nel sud dell'Albania. Il titolo dell'opera è lo stesso del nome del bar che, l'ex-studente di storia - Drini, gestisce a Torino. Tra le sue occupazioni spicca lo spaccio della droga. Il protagonista di Kubati suscita compassione, vista l'ingiustizia che subisce, l'ostilità di cui è destinatario, quello di Levani invece risulta piuttosto negativo. Comunque lo caratterizzano alcuni pregi, è deciso e intelligente ed ha tradito anche la propensione ad aiutare altri. Diversamente da Kubati, Levani non si concentra sulle difficoltà che gli immigrati fronteggiano nel paese d'adozione, li presenta invece come figure audaci, che non esitano a infrangere la legge e così il lettore si immerge in una ricca immagine della malavita albanese in Italia. Tale fattore rende particolare il romanzo „Toringrad” nel corpus prodotto da autori migranti provenienti dall'Albania, nel quale di solito l'accento viene posto sulla negatività che i loro connazionali sperimentano vivendo sull'altra sponda dell'Adriatico. In più opere si percepisce dunque una faccia avversa degli italiani, come si vede anche in Kubati. Le riflessioni dello scrittore sulla condizione degli stranieri sono di ampio respiro, non si limitano soltanto al contesto italiano, ma si estendono a quello europeo. L'autore evidenzia come gli abitanti dell'Occidente abbiano difficoltà nell'accettarli e non riescano a capire che sono sperduti e hanno bisogno di trovare l'altrimenti ${ }^{10}$ solo per continuare a vivere. Il principale ostacolo a una loro vita migliore, dice Kubati, non costituiscono confini fisici, ma quelli metaforici costruiti dagli stessi locali attraverso il rifiuto dell'altro.

L'aspetto più indigesto della questione mi sembrava il fatto che gli afroeuropei e gli euroafricani (o tutte le simili combinazioni), invece di avvicinarsi, si allontanavano inesorabilmente in questa danza di identità che vedeva, ancora una volta, i primi maggiormente e tragicamente svantaggiati. Era come se gli europei vietassero agli

\footnotetext{
${ }^{10}$ In Kubati il desiderio di trovare l'altrimenti caratterizza in modo particolare i giovani. Vista la specificità del periodo che attraversano, la visione del tempo che hanno ancora davanti a sé, la forza che possiedono, cercano posti favorevoli dove poter costruirsi una nuova vita. Tale ricerca non risulta dalla loro curiosità giovanile, ma dall'impossibilità di vivere in modo decente nel paese d'origine. A proposito del tema dell'altrimenti in Kubati, si consulti lo studio di S. Wright (2003-2004).
} 
altri di fuggire nel loro altrimenti, perché l'altrimenti europeo erano proprio loro. Una fisiologica esaltazione della culturodiversità auspicava la conservazione dei riservati naturali, in cui il solco del peggio era stato superato già da un pezzo ${ }^{11}$.

Agli occhi degli autoctoni gli immigrati sono sovente personaggi peggiori, sospettati dell'intenzione di commettere diversi reati. Tale loro atteggiamento campeggia in un'altra situazione problematica individuabile nel romanzo. Inserendola nella trama, Kubati tocca un tema di grande attualità in Italia, ossia la presenza di badanti straniere in questo paese. $\grave{E}$ opportuno accentuare che un'ampia visione della vita di una badante tipica viene delineata nel romanzo „Badante per sempre” (2015) di Ingrid Beatrice Coman, una scrittrice migrante di origine romena. Coman accenna in modo molto forte alle difficoltà affrontate dalla protagonista Magda, obbligata a lasciare la Romania in cerca di una vita migliore nel 1989, dopo il crollo della dittatura di Ceausescu e giunta in Italia per rimanervi stabilmente. Viene messo in risalto come sia sovente oggetto di diffidenza e ostilità degli anziani di cui si prende cura. Col tempo riesce ad acquistare la loro simpatia e costruirsi relazioni abbastanza serene con l'ambiente circostante. Comunque la vediamo sempre sospesa tra la nuova realtà e quella romena, sempre viva nei ricordi.

E risaputo che la professione di badante viene eseguita soprattutto dalle donne e Coman conserva questo paradigma. Nel romanzo in esame invece essa viene affidata a una figura maschile. Il protagonista di Kubati, per un lungo periodo di tempo, si occupa del cosiddetto nonno - un signore avanzato negli anni, malato e immerso nella solitudine. L'impiego è impegnativo ed esige una grande pazienza e determinazione, visti i profondi disturbi mentali che caratterizzano il vecchietto, ostacolano la comunicazione e sovente gli fanno perdere il contatto con il mondo. Tale suo stato dà luogo a malintesi, tensioni, giudizi sbagliati e qualche accusa. In più, il personaggio principale sarà colpito da uno stress intenso dopo la morte dell'uomo. La polizia lo sospetta di omicidio e perciò viene incarcerato. Uno dei motivi della sua carcerazione è proprio la provenienza straniera, che stavolta lo espone a un dolore enorme, capace di trasformarsi in depressione. Infatti è così afflitto da riflettere sulla propria morte, immagina come si comporterebbero le sue persone care se lui non vivesse più. Non teme di morire, si dimostra piuttosto indifferente nei confronti di tale eventualità. L'unico elemento che gli provoca angoscia e ribrezzo è la visione del proprio cadavere tratteggiata

\footnotetext{
${ }^{11}$ Kubati 2002, p. 149.
} 
nell'autocoscienza. Pare opportuno aggiungere che la sua reazione al corpo morto è conforme alla teoria di Julia Kristeva (2007), che lo considera come uno degli elementi che possono suscitare ripugnanza.

Il protagonista si immerge sovente nelle proprie riflessioni, dimostrandosi molto sensibile e compassionevole. Il pensiero della morte lo accompagna non solo in prigione. Scorgendo intorno a sé numerosi fenomeni negativi, viene invaso da un forte pessimismo che lo rende propenso a ritenere la morte come una fuga dal mondo in cui si è destinati soltanto a provare dolore, a subire conseguenze dell'ingiustizia e della povertà. Ecco una citazione che conferma quanto appena detto:

La morte è un momento della vita; un momento di trapasso che, quando uno fa una vita da verme, prima arriva, meglio $\grave{e}^{12}$.

Rimanendo incarcerato, si sente deluso e ingannato dalla sorte, i suoi atti di bontà, gli sforzi e il tempo dedicato a un uomo vecchio, non vengono apprezzati, ma al contrario, gli valgono delle accuse serie che suscitano la paura del futuro. Con la situazione ci inoltriamo nella visione di un mondo alla rovescia in cui il significato di ideali giusti si riduce in polvere e un individuo retto e cordiale, che si sacrifica per altri, è costretto a soffrire. Va aggiunto che in fin dei conti il protagonista viene liberato e non subisce gravi conseguenze penali. Tale esperienza però di sicuro influisce in modo negativo sulla sua psiche, diventa uno stigma interiore che gli riaffiora alla mente, al contempo è cosciente che non potrà mai redimersene in quanto risulta dalla provenienza geografica - una sua caratteristica che lo accompagnerà fino alla fine dei suoi giorni.

Diversamente da molti altri autori di origini straniere che si esprimono in italiano, e come esempio possiamo addurre Jadelin Gangbo $^{13}$, scrittore nato nella Repubblica del Congo, Kubati non accenna in modo tanto esplicito ai problemi linguistici e identitari dei migranti. Il suo protagonista comprende perfettamente i locali e in generale parla bene la loro lingua. Così risulta

\footnotetext{
${ }^{12}$ Kubati 2002, p. 86.

${ }^{13}$ Facendo riferimento a Gangbo, prendiamo in considerazione il suo racconto intitolato „Com è se giù vuol dire ko" (2005), in cui campeggia la figura di Aziz, un marocchino diciannovenne. Vista la sua origine straniera, è destinatario di atti di violenza e razzismo. Essi gli impediscono di identificarsi con il paese in cui vive. Il suo disagio interiore è molto serio. Da un lato indubbiamente desidera sentirsi italiano, dall'altro scopre che i locali lo considerano estraneo. La problematica identitaria che caratterizza l'opera è di sicuro legata alle esperienze dello stesso Gangbo. Lo scrittore è giunto in Italia da bambino all'età di 4 anni e ha subito un intenso processo di assimilazione attraverso l'educazione nelle scuole italiane e i contatti con i locali, si è costruito una vita normale e si considerava membro della società.
} 
giusto avanzare l'ipotesi che l'abbia imparata prima dell'arrivo. Di sicuro è sospeso tra la realtà del paese natale e quella del paese d'accoglienza, comunque non si immerge nelle riflessioni sulla sua appartenenza, ai propri occhi e anche agli occhi degli italiani, la sua identità straniera è però ben evidente. In futuro il suo legame identitario con l'Italia sarà più solido. Vi contribuiranno i vari avvenimenti positivi che hanno luogo durante il processo della sua integrazione.

\section{NEL NUOVO SPAZIO VITALE: \\ PROSPETTIVA ARMONIOSA}

Edward Said, un intellettuale palestinese di fama internazionale, nell'opera "Out of space" (2000), una raccolta di memorie, parla di numerosi aspetti della propria esperienza migratoria, che l'ha portato a vivere in paesi culturalmente molto diversi. Il lettore vi scopre la figura di un esule obbligato a confrontarsi con l'altro, con nuove culture, nuovi spazi e nuova gente, e perciò insicuro nella sua autocoscienza, pervaso dalla sensazione di spaesamento. Vista la situazione tumultuosa del popolo da cui proviene, Said dedica un'attenzione particolare al fenomeno dell'esilio politico e ne denuncia l'ingiustizia. Non è giusto, sembra affermare, che l'individuo abbandoni la terra natale per via delle decisioni delle autorità, sulle quali non è in grado di influire in nessun modo. Definendo l'esilio, Said rileva il rapporto conflittuale di chi lo vive con lo spazio. L'esule soffre in quanto rimane lontano da casa. In un'altra opera lo studioso scrive:

\footnotetext{
L'esilio è qualcosa di singolarmente avvincente a pensarsi, ma di terribile a viversi. E una crepa incolmabile, perlopiù imposta con forza, che si insinua tra un essere umano e il posto in cui è nato, tra il sé e la sua casa nel mondo ${ }^{14}$.
}

Dalla citazione risulta che Said considera l'esilio come un'esperienza decisamente ostile, un tipo di condanna che va affrontata e che può sembrare favorevole soltanto se non vissuta realmente. Fino al momento in cui non si realizza davvero, l'individuo può dunque delinearne una visione carica di positività. La vita nel paese straniero provoca un senso di mancanza, di perdita e di distacco dalla terra natale. Le riflessioni di Said sono parzialmente conformi alla trama del romanzo in esame. Il protagonista probabilmente lascia il paese d'origine per motivi politici. La sua provenienza

\footnotetext{
${ }^{14}$ Said 2008, p. 216.
} 
è sconosciuta, ma, se lo identifichiamo con l'autore, e tale approccio è ben fondato, possiamo avanzare l'ipotesi che giunga in Italia per fuggire dall'oppressività del regime comunista, dalle conseguenze disastrose della sua politica. Come detto, più elementi del suo spazio vitale, tra cui l'atteggiamento di alcuni locali, le difficoltà relative al contatto con nuovi territori, problemi economici, rendono deplorevole il suo stato e gli fanno riaffiorare alla mente che la sua vera casa è molto distante. Comunque tale prospettiva non caratterizza in modo completo la vita del personaggio principale, che in fin dei conti si integra nella realtà del paese ospitante. Il processo della sua integrazione si estende a più elementi. Un ruolo molto importante riveste il nuovo rapporto che instaura con lo spazio e i locali, trasformatosi col tempo in modo significativo e divenuto sereno.

\begin{abstract}
Iniziavo a rendermi conto che il mio approccio con gli spazi, le attività e le persone del mio nuovo ambiente era cambiato. Mi sembrava che loro mi accettassero meglio e che le mie fantasie si accomodassero più a loro agio nei locali, strade e quartieri. Anche un bar all'angolo del marciapiede della ennesima fermata del tram lasciava intravedere interni accoglienti per un apperitivo o serata tra amici ${ }^{15}$.
\end{abstract}

La narrazione applicata al romanzo rinsalda due piani temporali: il presente che riflette il soggiorno del protagonista in Italia e il passato che ingloba riferimenti al periodo che ha trascorso nel paese natio, compresi gli anni dell'infanzia. Attraverso tale tecnica i lettori prendono coscienza che la curiosità, il desiderio di cercare il nuovo e l'altro, caratterizzano la sua personalità fin da piccolo.

\begin{abstract}
Mi ero accorto della finestra quando ero bambino. (...) Appena sveglio guardavo la finestra per capire da quale parte ero, che tipo di giornata mi aspettava, che cosa mi era permesso, ecc... (...) Da quel momento in poi, cambiai molte finestre. Con un senso di meraviglia, mi alzavo ogni volta per iniziare un altro giorno e per scoprire dove e chi ero. (...) Sentivo, insieme all'inevitabile entusiasmo e curiosità che il nuovo mi suscitava, anche una forma di emorragia, un grande senso di perdita. (...) Avevo dentro, contemporaneamente, una grande quantità di emozioni che variavano dal dolore sempre acuto, dalla umida e profonda nostalgia, fino a un vertiginoso entusiasmo causato da tante nuove esperienze (...). Un sentimento di estraneità precedeva sempre il senso di meraviglia davanti alle tante finestre che, ininterrottamente, si aprivano sul mondo: fossero un libro, un ricordo, una fantasia, una persona, un amore o una nuova città $^{16}$.
\end{abstract}

Attraverso la metafora della finestra Kubati costruisce una tipologia interessante del migrante che appare come destinato a spostarsi e a scoprire territori ignoti. La sua permanenza nel paese ospitante potrebbe dunque

\footnotetext{
${ }^{15}$ Kubati 2002, p. 64.

${ }^{16}$ Ibidem, pp. 90-91.
} 
risultare anche dalla costruzione della sua psiche, dalla sua innata propensione alla curiosità, la quale lo spinge a rischiare e a prendere decisioni coraggiose.

La finestra marca il confine fra quello che conosce e l'altrove che si accinge or ora a esplorare e che lo porterà a vivere esperienze insolite. I sentimenti che prova nei loro confronti sono condizionati dal tempo. Inizialmente lo invade un senso di estraneità e timore, una volta immerso nel nuovo mondo però, se ne meraviglia. Il suo rapporto con lo spazio italiano ha lo stesso carattere. I continui spostamenti al suo interno, come sottolinea Chiara Mengozzi $^{17}$, gli consentono di abituarsi alla topografia della città. Le barriere fisiche, ossia le vie e i percorsi inesplorati prima, spariscono e ciò fa sì che possa muoversi liberamente e sentirsi più a proprio agio. Lo scrittore albanese pone l'accento sulla natura fluida della personalità umana, sulla sua capacità di subire trasformazioni. Lo stato d'animo del soggetto migrante risulta completamente condizionato dagli impulsi esterni di cui è destinatario. La conoscenza dello spazio rinsalda il legame con il paese ospitante e gli potrebbe provocare la sensazione di costituirne una parte, comunque non riesce a considerarlo proprio. Tale accostamento verso la dimensione che prima si dimostrava completamente estranea introduce senz'altro molta positività nel suo animo. Fiorangelo Buonanno analizza in modo scrupoloso il momento dell'arrivo del protagonista in Italia, i primi contatti con la sua infrastruttura, e lo definisce come una rinascita ${ }^{18}$. E ovvio che, giungendo in terra straniera, lui esprime il desiderio di raggiungere la felicità e distanziarsi dal passato. Di una sua rinascita si può parlare però solo quando il rapporto con lo spazio e i locali perde il carattere conflittuale.

Nel processo di integrazione che ci interessa in questa sede un ruolo importante svolge non solo la topografia della città, ma anche il microspazio in cui il personaggio principale ha deciso di dimorare, acquistando così la possibilità di avere un luogo dove poter sentirsi sicuro. Il suo proprietario Andrea - un insegnante precario di letteratura, un uomo di media età, aperto, privo di pregiudizi razziali, gli offre rifugio in quanto spinto da un sentimento di solidarietà con gli immigrati. Kubati lo presenta come un individuo intenzionato a lottare contro ogni forma di ingiustizia, a opporsi contro chi non rispetta i diritti umani. „In fondo prof. Andrea era principalmente un "protestante”. Bastava guardarlo in faccia e non avevi

\footnotetext{
${ }^{17}$ Mengozzi 2008.

${ }^{18}$ Buonanno 2016, p. 20.
} 
dubbi. Era questa la sua indole, la cui netta esistenza era la sua reale forza"19. Abbiamo a che fare con un cittadino italiano che tratta da figlio un giovane straniero, gli dà consigli pregevoli, modella il suo comportamento, aiuta a risolvere i problemi. Si presenta come un padre e un maestro molto intelligente, dal carattere retto, che possiede un grande sapere sul funzionamento del mondo, nonché la capacità di intuire i bisogni altrui. Una grande dose di bontà emana da Andrea e diventa un impulso emozionale che il personaggio principale riceve e manda ad altri. Il suo atteggiamento benevolo verso il prossimo, la prontezza ad assistere chi ne ha bisogno risultano dunque dalla positività di cui è destinatario, dalla sparizione delle barriere che lo limitavano vista la provenienza, tra cui il senso di inferiorità e la mancanza di fede nell'utilità delle proprie azioni. Fin dall'inizio la sua relazione con Andrea è piena di cordialità e simpatia e ha un impatto enorme sulla sua psiche, contribuisce a eliminare l'angoscia dall'autocoscienza, lo rende capace di agire e seguire l'esempio del maestro. Ciò è ben visibile quando scopre che Betti, una prostituta, è stata violentata ed è sola a soffrire e a lamentarsi in una delle stanze della casa. La grande preoccupazione che prova lo spinge ad aiutarla in qualche modo.

Notai sul suo viso, oltre ai già noti lividi in via di guarigione, dei nuovi, più grandi, più neri. Aveva uno sguardo blu scuro che brillava tra le lacrime e il dolore. I suoi lamenti non si articolavano. Sul collo c'erano graffi sparsi. (...) Andai in cucina e misi sul fuoco la caffettiera, alla quale aggiunsi polvere di caffè in abbondanza e poca acqua. (...) le feci bere della grappa, pensando che qualsiasi cosa forte in un senso o nell'altro, le avrebbe fatto bene. (...) Volevo chiamare polizia, carabinieri, il padre eterno, l'ospedale, prof. Andrea ${ }^{20}$.

Introducendo la figura di Andrea, Kubati trasmette un altro messaggio importante sulla società italiana contemporanea, conferma che tra i suoi membri si possono individuare non solo razzisti pieni di pregiudizi, ma anche soggetti propensi ad aiutare i migranti. Al contempo lo scrittore rileva la funzione assunta nel contesto migratorio dalla casualità, oppure meglio, dal destino. Di solito il migrante non sceglie la località che sia la meta del suo viaggio. $\mathrm{Da}$ un lato, giungendo in terra straniera, spera bene per il futuro, dall'altro dà la sua vita alle mani della sorte, corre il rischio di essere respinto, umiliato o rimpatriato.

La positività che va dalla nuova casa nella direzione del protagonista deriva da un'atmosfera singolare e amichevole che vi regna, creata dallo

\footnotetext{
${ }^{19}$ Kubati 2002, p. 122.

${ }^{20}$ Idem 2002, p. 93.
} 
stesso padrone e da alcune persone che sovente la frequentano. Sono personaggi emarginati la cui vita è imbevuta di problemi seri relativi soprattutto alla mancanza di mezzi economici e che, ciononostante, guardano con ottimismo al futuro. La casa diventa una scena su cui si interpretano i drammi interiori che vivono, ma anche un'oasi di pace, un riparo dove si condividono delle esperienze serene. Così viene costruita una dimensione che attesta come sia ibrido il carattere dell'esistenza umana, l'esistenza che di solito integra la negatività alla gioia. Il romanzo di Kubati riflette perfettamente la quotidianità di molti individui contemporanei imbevuta di momenti sia felici che mesti. Proprio a casa di Andrea il processo di integrazione su cui indaghiamo inizia a riguardare la prospettiva umana. Attraverso gli appuntamenti che vi avvengono, il soggetto migrante si accosta verso i locali, scopre le loro abitudini e riceve frequenti segni di simpatia.

Oltre a Betti, il vecchietto e Andrea, fra i personaggi centrali dell'opera in esame, lo stesso autore annovera la scenografa Claire e l'ausiliario di traffico Fabio $^{21}$. Un ruolo molto importante nella vita del protagonista svolge senz'altro Claire, una donna attraente di cui si innamora perdutamente e con cui intende costruire una relazione durevole. La capacità di innamorarsi svela il suo stato interiore, costituisce un sintomo del suo equilibrio psichico, è un altro segno che non prova più senso di inferiorità. L'amore fa sì che nelle sue riflessioni tocchi il tema del sesso e della sessualità. Così lo vediamo come un uomo tipico, desideroso di gustare il corpo femminile, propenso a immergersi nelle fantasie sessuali.

\begin{abstract}
In piscina incontrai Nicole Kidman che mi chiese se poteva attendere con me in acqua. Poi, parlando parlando, iniziai ad accarezzarla. Lei diventava sempre più bella. Gradualmente ci dimenticammo del diluvio in arrivo e l'incubo si trasformò in un sogno erotico dal quale non volevo più svegliarmi. Durante i baci, la Kidman si trasformò in Claire. E quando aprii gli occhi, diedi seguito alle fantasie, questa volta in modo più lucido. Restai nel letto per un'altra ora a fantasticare su Claire ${ }^{22}$.
\end{abstract}

Claire accetta l'amore del personaggio principale e ciò indubbiamente stimola le sue ambizioni, lo rende più sicuro di sé e spinge a conquistare nuove zone. Per tale motivo fa progetti per il futuro, esprime una forte intenzione di fare carriera come scrittore, dedica una parte considerevole del suo tempo all'elaborazione dei manoscritti e alla ricerca di un editore che sia disposto a pubblicarli. In più trova occupazioni che consentono di migliorare il suo status economico.

\footnotetext{
${ }^{21}$ Idem 2005, p. 60.

${ }^{22}$ Kubati 2002, p. 102.
} 
Il processo di integrazione in esame costituisce per Kubati un'occasione di presentare la situazione delle case editrici in Italia, nonché quella di aspiranti scrittori desiderosi di esordire. Il mercato dei libri si rivela monopolizzato da grandi compagnie, che promuovono autori affermati, e quelle piccole, a cui si rivolgono i migranti, hanno poche possibilità di sviluppo. In fin dei conti però il protagonista raggiunge lo scopo prefissato e pubblica un suo lavoro. Verso la fine della storia raccontata, il lettore assiste a una scena in cui ne viene preparata la prima presentazione. I suoi sentimenti in tale momento particolare sono un miscuglio di euforia e tensione. Da un lato prova gioia, dall'altro è preoccupato per i preparativi in corso. A questo punto vale la pena sottolineare che Kubati tocca il tema del migrante albanese che giunge in Italia anche nel romanzo intitolato „Va e non torna” (2000). Elton, l'alter ego dell'autore, non solo compone racconti, ma probabilmente, come osserva Chiara Mengozzi, scrive il romanzo che viene raccontato nella trama e che si concentra sulla vita di suo padre incarcerato a Spaç ${ }^{23}$. L'opera, come il testo analizzato, contiene molti elementi autobiografici. Oltre all'esperienza migratoria di Kubati, essi hanno forma di rimandi diretti alla situazione dell'Albania. Maria Grazia Negro ben nota che in „Va e non torna” la narrazione rinsalda due dimensioni temporali: quella del passato che riflette il periodo trascorso dal protagonista in terra natale, e quella del presente, ossia la sua vita in Italia $^{24}$. Sebbene riesca a integrarsi nella società italiana, vive sempre sospeso tra la nuova realtà e il paese d'origine, la cui immagine sovente gli riaffiora alla mente. Con i numerosi flashback, il lettore si inoltra in un quadro interessante dell'Albania dei tempi della dittatura comunista e del suo crollo definitivo all'inizio degli anni Novanta del Novecento.

Va posto in evidenza che gli autori migranti italofoni non di rado provengono da paesi del blocco ex-comunista e hanno trascorso una parte della loro vita obbligati a seguire le regole imposte dal sistema. Alcuni di loro desiderano denunciare l'ingiustizia subita e lo fanno adottando due prospettive. Nel romanzo „Va e non torna” Kubati ha un approccio molto individuale, anzì personale. I fatti che presenta riflettono in modo esplicito vari elementi della sua biografia. La stessa tendenza si percepisce nel testo di Gëzim Hajdari² ${ }^{25}$, il più famoso poeta migrante albanese in lingua italiana,

${ }^{23}$ Mengozzi 2012, p. 13.

${ }^{24}$ Mauceri e Negro 2009, p. 80.

${ }^{25}$ Hajdari è giunto in Italia all'inizio degli anni Novanta in fuga dalle minacce subite per via del suo atteggiamento reazionario verso le autorità comuniste e post-comuniste. Il primo libro dell'autore esce in Albania. La sua carriera fiorisce in esilio con le pubblicazioni nella lingua 
intitolato „Il poema dell'esilio” (2005). Hajdari fa ricorso alla narrazione in prima persona e ciò indubbiamente accosta la sua figura alla visione dell'Albania che presenta, ai tempi difficili del comunismo e del post-comunismo che ha sperimentato sulla propria pelle. L'autore si scaglia non solo contro i politici comunisti e tutti quelli che li hanno appoggiati, ma anche contro quelli che hanno preso il potere dopo il tracollo della dittatura e si sono dimostrati incapaci di eliminare l'ingiustizia dalla vita pubblica. Prendendo la decisione di recarsi in esilio, il poeta esprime la sua protesta contro le malefatte sempre presenti nel suo paese. La già menzionata Coman, nell'opera „Per chi crescono le rose” (2010), si serve invece di uno sguardo molto meno personale rispetto a Kubati e Hajdari. La narrazione è in terza persona e non è possibile identificare la scrittrice con una delle protagoniste. Coman fa ricorso alla storia di alcune figure della società della Romania comunista per denunciare la loro condizione. Vale la pena aggiungere che un'analisi interessante della realtà romena sotto la dittatura è presentata anche nella raccolta di liriche intitolata „Da solo nella fossa comune” (2006) di Viorel Boldis.

Chiusa questa breve parentesi, torniamo al romanzo „Va e non torna” e al suo parallelismo con l'opera in esame. Elton divide il suo tempo tra casa, lavoro, incontri con amici e con Elena, una ragazza di cui si innamora. L'atteggiamento che ha nei confronti delle donne si può paragonare a quello del protagonista di „M”. Ambedue gli uomini le vedono attraverso il prisma del sesso, tradendo così l'inclinazione al maschilismo. Un altro elemento in comune che caratterizza il loro soggiorno in Italia riguarda le esperienze vissute a contatto con i locali, da cui Elton non di rado riceve segni sia di ostilità che di simpatia. Tuttavia, quelli di simpatia dominano. Maria Grazia Negro scrive:

sono gli autoctoni a ricordare ad Elton la sua provenienza geografica e a manifestare verso i casi di emigrazione riuscita, che lui rappresenta, un atteggiamento alterno, di esaltazione sperticata da una parte e di disprezzo razzista dall'altro ${ }^{26}$.

La studiosa cita l'esempio di una situazione difficile e umiliante vissuta dal personaggio principale durante il lavoro in una pizzeria dove subisce un attacco razzista da parte del proprietario, che esprime così la sua fedeltà allo

d'adozione che padroneggia perfettamente. Hajdari è stato insignito di più premi, tra cui il prestigioso premio Montale nel 1997. Per un'analisi critica della sua produzione, si consultino: A. Gazzoni (2010) e S. Gianvito (2015).

${ }^{26}$ Mauceri e Negro 2009, p. 81. 
„stereotipo dell'albanese rozzo ed ignorante per coprire i suoi giochetti furbi di levitazione scorretta del conto" 27 .

\section{CONCLUSIONI}

Nel romanzo „M” Kubati delinea un quadro complesso della condizione del migrante giunto nel paese d'adozione, la quale dipende da fattori classificabili in due categorie: quella di fattori fissi e quella di fattori mobili oppure alterabili. La prima categoria comprende lo spazio della città dove inizia a vivere, il quale conserva la sua posizione fisica e va esplorato per muoversi decisamente. La seconda categoria invece ingloba l'atteggiamento degli autoctoni che sono organismi vivi, capaci di riflettere, di formulare giudizi e perciò molto diversi gli uni dagli altri.

Il soggetto migrante acquista un sapere rilevante sullo spazio e spostandosi riesce a non provare più paura per se stesso. I movimenti che compie diventano più audaci e sicuri. Tralasciati alcuni atti di razzismo, molti italiani lo trattano bene. Ciò di sicuro dipende dalla loro costruzione psichica individuale, dall'opinione positiva che esprimono sulla permanenza degli stranieri nel loro paese. Kubati ci presenta la figura di un migrante integrato nella società che comunque è ben cosciente della sua alterità, del suo status specifico in Italia, quello di un profugo, di uno sconosciuto. Nella sua autocoscienza lo accetta, la sua identità nazionale non si configura mai come un concetto problematico, e non vi dedica riflessioni profonde, più importante si dimostra la possibilità di acquisire mezzi per vivere, soddisfare le ambizioni professionali, soprattutto il desiderio di pubblicare il manoscritto elaborato, che diventa impellente dal momento che la sua vita si normalizza. Il protagonista trova più occupazioni che forse non gli danno gradimento, ma rendono possibile comprare cose necessarie, in più si innamora davvero e il sentimento viene corrisposto. Al suo equilibrio psichico contribuisce anche la pubblicazione del suo primo libro e il lettore può supporre che raggiunga la felicità.

Ambedue le categorie suddette di fattori sono condizionate dal tempo, dalla sua fluidità che porta cambiamenti significativi. Col tempo, rileva lo scrittore, la realtà si trasforma, diventa amichevole. Nel romanzo la migrazione non è uno stigma oppure una condanna, ma al contrario, essa consente di realizzare molti scopi e di sperare bene per il futuro.

${ }^{27}$ Ibidem 


\section{TO TRAVEL AND LIVE ELSEWHERE. ON THE EXISTENTIAL POSITION OF THE MIGRANT IN A FOREIGN COUNTRY IN „M” BY RON KUBATI}

\section{Summary}

Kubati's novel entitled "M", in which there is no shortage of autobiographical elements, draws on the experience of migration that the author partook in the early 1990s, having left his homeland for Italy. Where the story takes place is unspecified, though the reader is well aware that it is nowhere else but in Italy. The author takes advantage of his own, extensive knowledge and draws a very comprehensive picture of the migrant's existential position in a foreign land. This paper, comprising two main parts, aims to analyze that position, striving to delineate the nature of the phenomenon of migration. The first part is devoted to the problems that a migrant is forced to confront. Being lost in a new space, the daily experience of dearth of financial means and acts of racism exacerbate the sense of inferiority and lack of belonging. The second part discusses the process of the migrant's social integration. With time, the knowledge of the city becomes sufficient to move about with ease, while contacts with its native inhabitants are characterized by kindness and openness. The sojourn in a foreign country proves to be a positive experience, enabling the protagonist to achieve numerous goals and entertain an optimistic outlook on the future.

\section{Bibliografia}

Bregola D. 2002, Da qui verso casa, Roma.

Boldis V. 2006, Da solo nella fossa comune, Bologna.

Buonanno F. 2016, La percezione della città nella letteratura italiana della migrazione, Études romanes de Brno, pp. 17-30.

Coman I.B. 2015, Badante pentru totdeauna. Badante per sempre, Milano.

Coman I.B. 2010, Per chi crescono le rose, Milano.

Di Gianvito S. 2015, In balia delle dimore ignote. Saggi su Gëzim Hajdari, Nardò.

Dones E. 2007, Vergine giurata, Milano.

Gangbo J. 2005, Com è se giù vuol dire ko, AaVv, Italiani per vocazione Fiesole, pp. 137-186.

Gazzoni A. 2010, Poesia dell'esilio. Saggi su Gëzim Hajdari, Isernia.

Hajdari G. 2005, Il poema dell'esilio, Santarcangelo di Romagna.

Kristeva J. 2007, Potęga obrzydzenia. Esej o wstręcie, Kraków.

Kubati R. 2016, La vita dell'eroe, Nardò.

Kubati R. 2005, Alla ricerca dell'altrimenti „Va e non torna” e „M”, [in:] A. Gnisci (ed.), Allattati dalla lupa. Scritture migranti, Roma, pp. 49-65.

Kubati R. 2002, M, Nardò.

Kubati R. 2000, Va e non torna, Nardò.

Levani D. 2016, Toringrad, Santa Maria Capua Vetere.

Mauceri M.C., Negro M. G. 2009, Nuovo Immaginario Italiano, Roma. 
Mengozzi C. 2012, Scena interlocutoria e paradigma giudiziario nelle scritture italiane della migrazione, Between, pp. 1-22.

Mengozzi C. 2008, Città e modernità: nuovi scenari urbani nell'immaginario della letteratura italiana della migrazione, [in:] C. Gurreri, A. Jacopino, A. Quonadam (ed.), Moderno e modernità: la letteratura italiana, Roma, pp. 1-9.

Said E. 2000, Out of place, New York.

Said E. 2008, Nel segno dell'esilio. Riflessioni, letture e altri saggi, Milano.

Sorina M. 2006, Voglio un marito italiano. Dall'est per amore?, Vicenza.

Spanjolli A. 2012, I nipoti di Scanderbeg, Nardò.

Spanjolli A. 2011, La sposa rapita, Nardò.

Spanjolli A. 2006, La Teqja, Nardò.

Wright S. 2003-2004, „Va e non torna” e „M”, ovvero la poetica dell'altrove e dell'altrimenti nei romanzi di Ron Kubati, NEMLA Italian Studies, pp. 113-133. 